\title{
WARM-UP EFFECTS IN THE LEARNING OF DISCRETE MOTOR SKILLS ${ }^{1}$
}

\author{
Craig A. WRISBERG, ${ }^{2}$ Alan W. SALMONI, ${ }^{3}$ and Richard A. SCHMIDT ${ }^{4}$ \\ Dept of Physical Education, University of Michigan, Ann Arbor, U.S.A.
}

Received January 1975

\begin{abstract}
Three experiments were conducted to determine the influence of warming-up activities on performance and on learning in two discrete motor tasks. During initial trials on the criterion task, $S \mathrm{~s}$ were given various types of warming-up activities having an activity-set that was either the same as or different from the criterion task. Test trials followed in which all groups practiced the criterion task only. No significant differences in performance on the criterion task were found regardless of the appropriateness of the warming-up activity during initial trials. The results suggested that warming-up properties are not a factor in original learning of discrete motor skills.
\end{abstract}

The initial post-rest decrement in performance on a task that has been learned previously has generally been attributed to $S$ 's need to 'warm up', and is characterized by an extreme brevity, rarely lasting for more than the first few trials following rest. Investigations of this phenomenon in motor skills have usually involved tests of the 'activityset' hypothesis, first proposed by Nacson and Schmidt (1971). According to this view, warm-up decrement (WU) is the result of the loss of a 'generalized' readiness (activity-set) for a given class of tasks which is supportive of the goal response yet 'neutral' in that it lends no increments of habit strength to the goal response. More specifically this hypothesis predicts that retention of the goal response should be

1 Requests for reprints should be sent to Craig A. Wrisberg, Division of Health, Physical Education, and Recreation, Virginia Polytechnic Institute and State University, Blacksburg, Virginia 24061.

${ }^{2}$ Now at Virginia Polytechnic Institute and State University, Blacksburg, Virginia 24061.

${ }^{3}$ Now at the University of Waterloo, Waterloo, Ontario.

4 Now at the University of Southern California, Los Angeles, California 90007. 
greater after performance of an appropriate warming-up task interpolated at the end of a rest period than following trials on an inappropriate neutral activity. Regardless of the apparatus or limb used, the warming-up task must be of the same class as the criterion task if WU is to be reduced or eliminated. How narrow this class of activities is is not specified, but it is arbitrarily assumed that the activities in a class possess certain response requirements (such as accurate arm positioning over trials with knowledge of results, KR), and that any positioning response with these requirements (e.g., arm positioning, leg positioning, etc.) is a member of the class.

Recent evidence from Schmidt and his associates (Nacson and Schmidt 1971; Schmidt and Nacson 1971; Schmidt and Wrisberg 1971) clearly indicates that post-rest WU in several classes of tasks can be reduced by appropriate neutral interpolated activities. However, only one study has been reported in which there has been a direct attempt to measure the effects of warming-up activities on the learning of motor skills. Hanilton and Mola (1953) evaluated the effect of practice on five different fïnger mazes either 24 hours or immediately preceding initial trials on a criterion maze. Since positive transfer was found for both groups, it was reasoned that the warm-up mazes contributed increments of habit strength rather than neutral warming-up properties to the criterion maze. Thus, inferences regarding the effect of warmingup in motor learning remain dubious.

The present series of experiments was designed to investigate whether an appropriate warming-up activity practiced during initial trials on a criterion motor task would facilitate learning of the task. A paradigm was used in which all $S$ s received initial trials on the criterion task with the principal independent variable of interest being whether $S$ was warmed up prior to or during these trials. After a rest, $S$ s were then given test trials on the criterion task only, to determine whether warming-up activities had affected learning.

\section{Experiment I}

\section{Method}

Subjects

Right-handed male and female graduate and undergraduate students at the University of Michigan $(N=40)$ served as $S$ s in Ixp. I. None was paid for his or her services. 


\section{Right-hand task apparatus}

A hand-grip device modified from a hand dynamometer was fastened to a $91.4 \mathrm{~cm} \mathrm{long}, 5.1$ $\times 30.5 \mathrm{~cm}$ wooden stand bolted to a desk $81.3 \mathrm{~cm}$ high. A cable connected the hand grip to a strain gauge which when stretched produced a signal which was amplified by a four-channel amplifier and output on a meter. The force was scaled in units from 0 to 100 with a reading of ' 50 ' representing a force of $15 \mathrm{lb}$. Thus, each scale unit was equivalent to $0.3 \mathrm{lb}$ of force.

\section{Left-hand task apparatus}

A cable running through four pulleys connected a handle to the same strain gauge as used in the right-hand task. Running down the front of the desk to a pulley on the floor, the cable was directed horizontally to the rear of the desk. From there two more pulleys directed the cable diagonally upward to a final pulley at the top rear of the desk which sent it to the strain gauge. The handle and hand-grip device were located so that $S$ could operate them both from the same seated position.

\section{Procedures}

Ss were randomly assigned to one of two conditions with the restriction that each condition have $20 \mathrm{Ss}$. Following a description of the right- and left-hand tasks, $S$ was given one familiarization trial on the right-hand task, squeezing slowly and carefully and observing the response of the meter. The meter was then turned so that it could be viewed only by $E$ and five practice trials on the right-hand task (at a different force from that used in the experiment) were given to screen those $S$ s unable to squeeze to within \pm 10 units of the practice force (70 units). If there were no questions, the experiment was begun after a one-minute rest.

The command 'Ready' was always followed in 2 sec by 'Squeeze' for the right-hand task. When $S$ felt he had achieved the required force, he said 'There', holding the force until $E$ recorded the score and reported it to $S$ in units. Between trials $(15 \mathrm{sec}) S$ relaxed his hand completely, waiting for the next 'Ready' command in response to which he engaged the apparatus and removed any slack in the cable. All $S$ s received 5 initial right-hand trials, a rest of $10 \mathrm{~min}$, and then 10 test trials on the right-hand task.

The principal independent variable was the temporal location of a 5-trial block of left-hand warming-up trials during the 8-min interval prior to initial right-hand trials. The activity-set condition (AS) received 5 left-hand trials at the end of this interval while the control condition (C) received 5 left-hand trials at the beginning. Since activity-set reinstatement has been found to be negated when as many as $40 \mathrm{sec}$ are interpolated between the warming-up activity and practice on the criterion task (Schmidt and Nacson 1971), only Condition AS which received left-hand practicc immediately prior to initial right-hand trials was expected to profit from the warming-up activity. In both conditions, the warming-up force to be estimated with the left-hand was $5 \mathrm{lb}$ (i.e., 20 units) and $\mathrm{KR}$ was given in units. By learning to estimate a specific force with KR but without vision of the meter, $S$ was performing a task with the same activity set as the right-hand task but, as has been demonstrated by Nacson and Schmidt (1971), is neutral with respect to the right-hand task habit since it involves the opposite limb and different musculature.

Preceding each block of trials on either task $S$ s were instructed as to the force they should be attempting to estimate. Although performance on the right-hand task was the principal measure of interest, $S$ s were told that they should be trying to learn both right- and left-hand 
forees. This precaution seemed important for two reasons. First, in order to make inferences about learning it was important that a learning set be instated. Since the warming-up effect during initial learning was the issuc, a learning set could not be instated through accumulated experience (e.g., Harlow 1949). Therefore instructions to learn were reasoned to be the primary way of promoting the learning set. Furthermore, in light of an investigation by Adans (1955) which revealed post-rest decrement in rotary pursuit performance to be reduced only for $S$ s foreed to attend to a secondary task during the interpolated period, it was reasoned that by equally emphasizing right- and left-hand tasks in the present experiment, an accurate estimate of the effects of warming-up activities on learning could be obtained.

\section{Results}

Table 1 summarizes variable error (VE) and constant error (CE) for five-trial blocks during both initial and test trials on the right-hand task. While all $S$ s demonstrated considerable performance gains with practice, differences between groups were small for both initial and test trials. On initial trials, the Conditions effect failed significance for both $\mathrm{CE}$ and $\mathrm{VE} \cdot F(1,36)=$ 1.35 and $F(1,36)=1.81, p>0.05$, respectively. The Conditions comparison on the test trials yielded similar results, $F(1,36)=1.35$ and $F(1,36)=0.11, p>0.05$, respectively, thus failing to provide support for the prediction that an appropriate neutral warming-up activity practiced immediately prior to initial performance would facilitate learning of the task. There was some indication that temporal location of the left-hand task in the interval preceuing initial trials affected CF. While not significant, Condition AS demonstrated a 1-th greater positive CF (i.e., overshooting) than the control condition on initial trials.

One possibility for the lack of differences was that the warm-up effect did not persist throughout the entire block of initial trials. Recent evidence (Godwin and Schmidt 1971) has suggested that a performance variable (e.g., fatigue) may also affect learning if manipulated vigorously during pre-rest practice. With this in mind, Experiment II was conducted using a paradigm in which $S_{s}$ were warmed-up prior to each initial trial on the right-hand task in order to minimize the possibility that the benefits of warming up might dissipate before the block of initial trials was completed.

Table 1

VE and CE (1b.) for the 5-trial blocks of initial (block 1) and test (blocks 2 and 3) trials on the right-hand task in Experiment $I$.

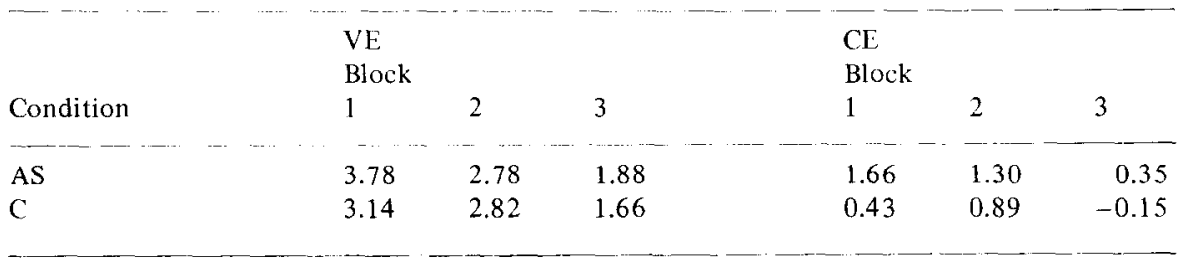




\section{Experiment II}

\section{Method}

\section{Subjects}

Right-handed male and female graduate and undergraduate students at the University of Michigan $(N=45)$ served as $S s$ in Exp. II. None had had previous experience in a forceestimation experiment and none was paid for his or her services.

\section{Apparatus and task}

Both apparatuses were identical to those used in Exp. I. The commands to $S \mathrm{~s}$, the target locations, and the nature of KR following each trial were the same as well.

\section{Procedures}

$S$ s were randomly assigned to one of three conditions with the restriction that each condition have $15 \mathrm{Ss}$. The following features distinguished Exp. II from Exp. I: (i) The initial right-hand trials were increased from 5 to 25 in order to give $S$ s more opportunities to delineate the nature and demands of the task; (ii) test trials on the right-hand task were increased from 10 to 15 ; (iii) the number of conditions was increased from 2 to 3 with a control condition (C) given no warm up during initial trials and a maximum pull condition (MP) receiving left-hand trials which required a maximum effort; and (iv) 3 trials on the left-hand task (Conditions MP and AS only) preceded each of the 25 initial right-hand trials. It was reasoned that in addition to instating an inappropriate activity-set for the right-hand task (i.e., maximum pulls rather than slow, careful pulls to a designated level), the MP Condition would provide a case wherein decreased performance on initial trials, and hence decreased learning on test trials, of the right-hand task would be evidenced. As in Exp. I, Ss were told that both right- and left-hand forces were equally important. Following initial trials and a 10 -min rest, all $S$ s were given 15 test trials on the right-hand task with a $15-\mathrm{sec}$ intertrial interval filled only by rest; these trials served as the measure of learning.

\section{Results}

$\mathrm{VE}$ and $\mathrm{CE}$ for blocks of initial and test trials on the right-hand task are summarized in table 2. As in Exp. I, there were no significant differences in $\mathrm{CE}$ among Conditions either on initial or test trials, $F(2,44)=1.44$ and $F(2,44)=1.26, p>0.05$, respectively. VE on trials $6-25$ revealed significant differences among Conditions, $F(2,44)=7.30, p<0.05$, but the ordering of $\mathrm{VE}$ was contrary to that expected. Of the three conditions, $\mathrm{C}$ displayed the lowest $\mathrm{VE}, \mathrm{MP}$ was intermediate, and AS was highest. Therefore, rather than providing $S$ s an advantage, it appeared as though activity-set instatement (AS) induced decrement in force-estimation per- 
Table 2

VE and CE (lb.) for the block of initial (10 trials) and test (15 trials) trials on the right-hand task in Experiment II.

\begin{tabular}{lllll} 
& VE & \multicolumn{1}{l}{ CE } \\
Condition & Block & \multicolumn{2}{l}{ Block } \\
\hline AS & 1 & 2 & 1 & 2 \\
C & 3.51 & 2.87 & 0.46 & 0.61 \\
MP & 2.24 & 2.00 & -0.26 & 0.23 \\
\hline
\end{tabular}

formance. On the block of test trials, there was no significant difference in VE among Conditions, $F(2,44)=2.17, p>0.05$, indicating that the effect found during initial trials was only temporary.

While CL for Condition MP during initial trials was not significantly greater than that of the other groups, an analysis of the first five right-hand trials revealed that $\mathrm{CE}$ was an increasing function of the amount of force exerted, (i.e., $\mathrm{C}=3.93 ; \mathrm{AS}=10.96 ; \mathrm{MP}=12.76) F(2,44)=$ $3.03, p=0.05$. This suggested that at least on carly trials of force estimation learning intensity as well as temporal placement of the warming-up activity may be a variable affecting $C E$.

Experiment III was conducted to determine whether the failure of appropriate warming-up activity to influence learning in Exp. I and II was limited to tasks of force estimation. In Exp. III, a movement-speed task was used.

\section{Experiment III}

\section{Method}

\section{Subjects}

Right-handed male and female graduate and undergraduate students at the University of Michigan $(N=68)$ served as $S \mathrm{~s}$ in Exp. III. None was familiar with the apparatus and none was paid for his or her services.

\section{Right-hand task}

The task was the Sigma task (see Godwin and Schmidt 1971; Schmidt and Wrisberg 1971), so named because of the $\sigma$-shaped movement of the hand which was required. It consisted of a handle (11 cm high and $2.6 \mathrm{~cm}$ thick) mounted vertically on the end of a horizontal $22 \mathrm{~cm}$ aluminum lever. The opposite end of the lever was attached to a vertical axle which ran in a bearing firmly mounted in a wooden box-like structure $(30 \times 49 \times 10 \mathrm{~cm})$ so that the lever was free to rotate in the horizontal plane. A sturdy metal stop positioned on the edge nearest $S$ 
allowed movement through only $344 \mathrm{deg}$. On another edge of the box $43 \mathrm{~cm}$ from the axle was a padded masonite barrier $(10 \mathrm{~cm}$ square) fastened with a hinge so that a slight tap was sufficient to cause the barrier to fall. The box was mounted securely to a large table $76.2 \mathrm{~cm}$ high.

In this task, $\dot{S}$ began with the apparatus in the starting position, with the handle against the stop and the barrier upright. $S$ was seated in a straight- backed wooden chair positioned so that the handle, when against the stop, was at his midline. On the command 'Ready' from $E, S$ grasped the handle firmly. On the command 'Go' which always came 2 sec later, $S$ moved the handle clockwise (i.e., initially to his left) $344 \mathrm{deg}$. until it struck the metal stop, then reversed his direction to return the handle to the starting position. When the handle struck the stop again, he released it and moved his hand to the right $43 \mathrm{~cm}$ to knock over the padded barrier. The task was to make this defined movement as quickly as possible. Moving the handle to the left initially started a $0.1-\mathrm{sec}$ timer (Standard, Type S-1,6-V. dc clutch) and knocking over the barrier stopped it; thus, the score was the total time required to complete the defined movement consisting of two circular phases and a linear phase. Reaction time was not measured.

\section{Left-hand movement task}

The apparatus consisted of a wooden board $(46 \times 60 \times 2.5 \mathrm{~cm})$ mounted on the table to $S$ 's left of the right-hand apparatus. Mounted on the surface were (a) a start button which operated a microswitch, (b) a 'dummy' doorbell-type push button, and (c) a $10 \mathrm{~cm}$ square masonite barrier attached with a hinge. This task, demonstrated by Schmidt and Wrisberg (1971) to have an activity-set similar to the Sigma task, also required a rapid limb movement. $S$ was seated in the same chair but was rotated $90 \mathrm{deg}$. to his left so that he was facing the left-hand task apparatus. On the command 'Ready' $S$ depressed the start button with his left index finger, and $2 \mathrm{sec}$ later on the command 'Go' $S$ removed his finger from the start button, moved forward to press the dummy button, and then to the right to knock over the barrier, the movement being done as quickly as possible. Releasing the start switch started the timer and knocking over the barrier stopped it; thus, the score was the total time to complete the two-part movement. As in the right-hand task, reaction time was not measured.

\section{Left-hand accuracy task}

On a $21.6 \times 17.9 \mathrm{~cm}$ piece of paper were mimeographed six small tracing mazes $5 \mathrm{~cm}$ long with a track width of $0.32 \mathrm{~cm}$. Each maze was identical, requiring five directional changes for completion. The mazes were arranged in two rows with three mazes in each row. This task required careful left-hand tracing. Ss performing this activity were rotated $90 \mathrm{deg}$. to their left as in the left-hand movenent-speed task. On the command 'Ready' $S$ grasped a pencil $9 \mathrm{~cm}$ in length with a clenched left fist, and placed the pencil at the beginning of the first maze. On the 'Go' command which always followed 'Ready' by 2 sec, $S$ began navigating the maze attempting to stay within the lines. $S$ s were told that error-free completion of the maze was worth 5 points, error-free incompletion was worth 3 points, and error trials (going outside the boundaries) were worth 0 points. 


\section{Procedures}

$S$ s were randomly assigned to one of four conditions with the restriction that each condition have $17 \mathrm{Ss}$. On Day I, Conditions AS (left-hand movement-speed task) and AC (left-hand accuracy task) received 6 trials on their respective warming-up tasks prior to each of 10 initial right-hand Sigma trials. A long-rest control condition (LR) rested during the 1 min prior to each initial trial on the Sigma task while a short-rest condition (SR) received an unfilled intertrial interval of $15 \mathrm{sec}$. $\mathrm{KR}$ in the left-and right-hand movement-speed tasks was given in $0.01 \mathrm{sec}$ while in the left-hand accuracy task it was given in points. As in Exp. I and II, Ss were told that they should be trying to learn both right- and left-hand tasks. On Day II, all $S$ s received 10 Sigma test trials separated by a $15-\mathrm{sec}$ intertrial interval filled only with rest. These Day II right-hand trials provided a test of the effect of Day I treatments on learning. If an appropriate warming-up activity was a factor during initial right-hand trials, then Condition AS should have demonstrated superior performance on Day II.

\section{Results}

Sigma-task movement times for 10-trial blocks of initial and test trials are shown in table 3. Both the Conditions effect and the Conditions by Trials interaction for Day 1 performance failed significance, $F(3,64)=0.16$ and $F(27,576)=1.32, p>0.05$, respectively. On Day II with all conditions switching to the same right-hand task practice, lack of differences persisted both for Conditions $F(3,64)=0.92, p>0.05$, and Conditions by Trials, $F(27,576)=1.33, p$ $>0.05$, lending no support to the idea that in a class of skills requiring high rates of limb movement speed, practice on an appropriate warming-up activity prior to each initial trial on the criterion task will aid learning.

Table 3

Movement time (sec.) for 10-trial blocks of initial (block 1) and test (block 2) trials on the right-hand task in Experiment III.

\begin{tabular}{lll} 
& Block & \\
Condition & 1 & 2 \\
\hline AS & 1.48 & 1.23 \\
AC & 1.46 & 1.21 \\
LR & 1.50 & 1.21 \\
SR & 1.52 & 1.33 \\
\hline
\end{tabular}

\section{Discussion}

The obvious result of the present series of investigations is that opposite-hand warming-up prior to or during initial trials on a discrete motor task does not enhance learning. In all three experiments, condi- 
tions receiving appropriate warming-up activities during initial trials on the right-hand task failed to demonstrate a significant superiority on test trials separated by rest only.

The dependent measures used suggested, however, that warming-up activities were not entirely without effect. In Exp. II, the significantly greater VE for condition AS on initial right-hand trials indicated the possibility that attention to the demands (e.g., KR) of more than one task might be a critical variable. Since these $S$ s performed alternately on left- and right-hand tasks, it is reasonable to assume that full attention may have been diverted from the right-hand task thereby negating some of the positive effect of warming-up.

In Exp. I and II in which a task of force estimation was used, there were consistent $\mathrm{CE}$ trends during initial right-hand trials. The more intense the warming-up activity (Exp. II) and the closer its proximity to performance on the right-hand task (Exp. I), the greater was the positive shift in CE. Thus, it appeared that during initial right-hand force-estimation trials in Exp. I and II, opposite-hand warming-up activity shifted $\mathrm{CE}$ on the criterion task in a way similar to same-hand interpolated activity in the motor short-term memory experiments of Pepper and Herman (1970).

In conclusion, the results of the present series of experiments seem to indicate that warming $S$ s up prior to or during initial trials on a criterion task will not facilitate learning of the task. Such findings are opposite those of earlier studies which used already-learned criterion tasks and reported temporary reductions in post-rest decrement (i.e., WU) when warming-up activities were practiced at the end of interpolated rest intervals (Nacson and Schmidt 1971; Schmidt and Nacson 1971; Schmidt and Wrisberg 1971). Thus, it appears that while warming-up properties are a factor in well-learned tasks, they are without effect in the acquisition of motor skills.

\section{References}

Adams, J. A., 1955. A source of decrement in psychomotor performance. J. Exp. Psychol. 49, 390-394.

Godwin, M. A. and R. A. Schmidt, 1971. Muscular fatigue and discrete motor learning. Res. Quart. 42, 374-382.

Hamilton, C. E. and W. R. Mola, 1953. Warm-up effect in human maze learning. J. Exp. Psychol. 45, 437-441. 
Harlow, H. F., 1949. The formation of learning sets. Psychol. Rev. 56, 51-65.

Nacson, J. and R. A. Schmidt, 1971. The activity-set hypothesis for warm-up decrement. J. Motor Behav. 3, 1-16.

Pepper, R. L. and L. M. Herman, 1970. Decay and interference effects in the short-term retention of a discrete motor act. J. Exp. Psychol. (Monograph Supplement), 83, No. 2, Pt. 2, 1-17.

Schmidt, R. A. and J. Nacson, 1971. Further tests of the activity-set hypothesis for warm-up decrement. J. Exp. Psychol. 90, 56-64.

Schmidt, R. A. and C. A. Wrisberg, 1971. The activity-set hypothesis for warm-up decrement in a movement-speed task. J. Motor Behav. 3, 318-325. 